\title{
Studies on Spectral Interference of Neodymium on Analytes in Trace Metallic Impurity Analysis of Neodymium Matrix Using CCD-based ICP-AES
}

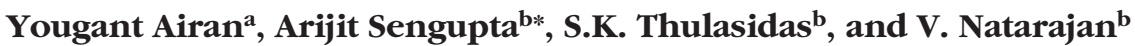 \\ ${ }^{a}$ Chemistry Department, Hindu College, University of Delhi, Delhi, India \\ ${ }^{b}$ Radiochemistry Division, Bhabha Atomic Research Centre, Trombay, Mumbai, India
}

\section{INTRODUCTION}

Lanthanides are electron-rich rare earth elements (REEs) and form an overlap contour of spectral lines which is the main source of error in atomic emission spectroscopy (1-5). REEs are widely used in areas of industry, agriculture, and modern technology, e.g., as a growth promoter fertilizers in agriculture, as a catalysts in oil refining, as additives in new ceramic materials and special steels, etc. (6-9). Neodymium is the raw material used in high-strength permanent magnets (Nd-B-Fe), making it less expensive than samarium-cobalt permanent magnets (10). Many investigators have used inductively coupled plasma atomic emission spectrometry (ICP-AES) as the powerful technique to determine REEs in geological samples (11-16). Capacitive coupled device (CCD)based ICP-AES is a highly sensitive method with a long dynamic range (17-18), has the capability to determine even trace amounts of the analytes (19-20), and has been used for over a decade (21-22). It also proved to be a better method than other earlier methods like neutron activation analysis (23-24) and isotope dilution mass spectrometry (25). A high temperature plasma $(6000-8000 \mathrm{~K})$ is used as the excitation source which minimizes matrix effects, prevents the formation of polyatomic species during the excitation process, which in turn produces adequate sensitivity and increases the detection limits for many elements (26). Before the

\footnotetext{
*Corresponding author.
}

E-mail: arijita@barc.gov.in

\section{ABSTRACT}

Due to the emission-rich spectra of all rare earth elements, they are expected to interfere in the determination of analytes at trace levels, especially when any of them is present as the matrix. The present paper deals with the study on the spectral interference of neodymium on 28 different analytes, including some rare earth elements $(\mathrm{Ag}, \mathrm{B}, \mathrm{Ba}, \mathrm{Ca}, \mathrm{Cd}$, $\mathrm{Co}, \mathrm{Cu}, \mathrm{Fe}, \mathrm{Mg}, \mathrm{Na}, \mathrm{Sr}, \mathrm{Zn}, \mathrm{Gd}$, $\mathrm{Lu}, \mathrm{Pb}, \mathrm{In}, \mathrm{Ce}, \mathrm{La}, \mathrm{Pr}, \mathrm{Ga}, \mathrm{Ni}, \mathrm{Al}$, Tl, Dy, Bi , Li, Mn, and Cr). This involves identification of the least interfered analytical lines of these elements, determination of correction factors, the tolerance level of $\mathrm{Nd}$, and the analytical performance of all the analytical lines of the elements in the presence of a Nd matrix. Since the extent of the spectral interference depends on the concentration of the interfering elements, it necessitates the development of ICP-AES-based methods for the determination of $\mathrm{Nd}$ which revealed that the $\mathrm{Nd} 401.225-\mathrm{nm}$ line is the best analytical line for Nd.

Based on the above study, a simple and rapid methodology was developed for the determination of these analytes at trace levels in the presence of a $\mathrm{Nd}$ matrix without any chemical separation. The method was validated using synthetic samples prepared from commercially available standard reference materials of the individual elemental solutions. advancements of multichannel detectors, photomultiplier tubes (PMT) were used as the detector with the major drawback of it being a single-channel detector which leads to a drastic waste of information, even when several detectors are set up in the polychromator. The multichannel detectors used with commercially available ICPAES systems based on charge transfer technology, such as the 2-D CCD detector or an association of linear CCD arrays, currently provide acquisition of the entire UVvisible spectrum: full flexibility in analytical line selection, use of several lines of the same element to extend the dynamic range, use of a large number of lines of the same element to improve accuracy and to verify possible matrix effects or spectral interferences, qualitative analysis and fast diagnostic. In addition to true simultaneous measurements, it offers speed of analysis, time correlation between lines of different elements to improve repeatability by internal standardization, and time correlation between line and adjacent background intensities to improve the limit of detection and the limit of quantitation (27-30).

Using the CCD-based ICP-AES instrument, the spectral interference of neodymium was studied for 28 analytes: $\mathrm{Ag}, \mathrm{B}, \mathrm{Ba}, \mathrm{Ca}, \mathrm{Cd}, \mathrm{Co}$, $\mathrm{Cu}, \mathrm{Fe}, \mathrm{Mg}, \mathrm{Na}, \mathrm{Sr}, \mathrm{Zn}, \mathrm{Gd}, \mathrm{Lu}, \mathrm{Pb}$, In, Ce, $\mathrm{La}, \mathrm{Pr}, \mathrm{Ga}, \mathrm{Ni}, \mathrm{Al}, \mathrm{Tl}, \mathrm{Dy}, \mathrm{Bi}$, $\mathrm{Li}, \mathrm{Mn}$, and $\mathrm{Cr}$. A simple and rapid method was developed for the trace level determination of these analytes in the presence of a Nd matrix since they are difficult to separate from $\mathrm{Nd}$ due to their similar chemistry. 


\section{EXPERIMENTAL}

\section{Instrumentation and Operating Conditions}

The analysis was carried out using an ARCOS inductively coupled atomic emission spectrometer (ICP-AES) (Arcos Industries, Germany), with argon plasma as the excitation source and a capacitive coupled device (CCD) as the detector system. The instrumental specifications and optimized operating conditions are summarized in Table I (30). The cross-sectional view of the detector system is given elsewhere (29). The resolution of the present instrument was documented as $0.01 \mathrm{~nm}$ for the wavelength range of $130-450 \mathrm{~nm}$, while for $450-800 \mathrm{~nm}$ it was $0.02 \mathrm{~nm}$. The resolution of the instrument plays an important role to resolve two closely spaced lines. The above resolution quoted by the manufacturer is one of the highest resolutions reported for CCD-based ICP-AES. The instrument with higher resolution can serve the purpose only when the lines are not resolved due to optical reasons only. But most of the time, even using the instrument with the highest possible resolution cannot resolve the overlapped emission lines. This case becomes more dominant when the interest is to determine the interfered analytes at trace levels in the presence of an interfering matrix.

TABLE I

Specifications and Operating Conditions of ICP-AES

\begin{tabular}{ll}
\hline Instrumental Specifications & \\
\hline Optical design & Paschen-Runge mounting, circular design \\
Focal length & $750 \mathrm{~mm}$ \\
Grating & Holographic \\
Groove density & 1800 grooves/mm (1) \\
& 3600 grooves $/ \mathrm{mm}(2)$ \\
Wave length range & $130-800 \mathrm{~nm}$ \\
Entrance slit width & 15 microns \\
Resolution (FWHM) & $0.01 \mathrm{~nm}$ from $130-450 \mathrm{~nm}$ \\
& $0.02 \mathrm{~nm}$ from $450-800 \mathrm{~nm}$ \\
Thermal regulation & Controlled to $30 \pm 1{ }^{\circ} \mathrm{C}$ \\
Frequency & $27.12 \mathrm{MHz}$ \\
Pump & Dual channel peristaltic pump (12 roller) \\
Detector & Linear arrays of CCD (3648 pixels/array) \\
Nebulizer & Cross flow nebulizer \\
ICP torch & Demountable, radial viewing \\
\hline Operating Conditions & \\
\hline Coolant flow & $13 \mathrm{~L} / \mathrm{min}$ \\
Auxiliary flow & $0.8 \mathrm{~L} / \mathrm{min}$ \\
Nebulizer gas flow & $0.8 \mathrm{~L} / \mathrm{min}$ \\
Total time of measurement & $28 \mathrm{~s}$ \\
Pump speed & $30 \mathrm{rpm}$ \\
Nebulizer pressure & $3 \mathrm{bars}$ \\
Pressure on manometer & $6 \mathrm{bars}$ \\
RF power output & $1.2 \mathrm{~kW}$ \\
Reflected power & $<10 \mathrm{~W}$ \\
Input power & $230 \mathrm{~V}$ AC \\
\hline & \\
& \\
&
\end{tabular}



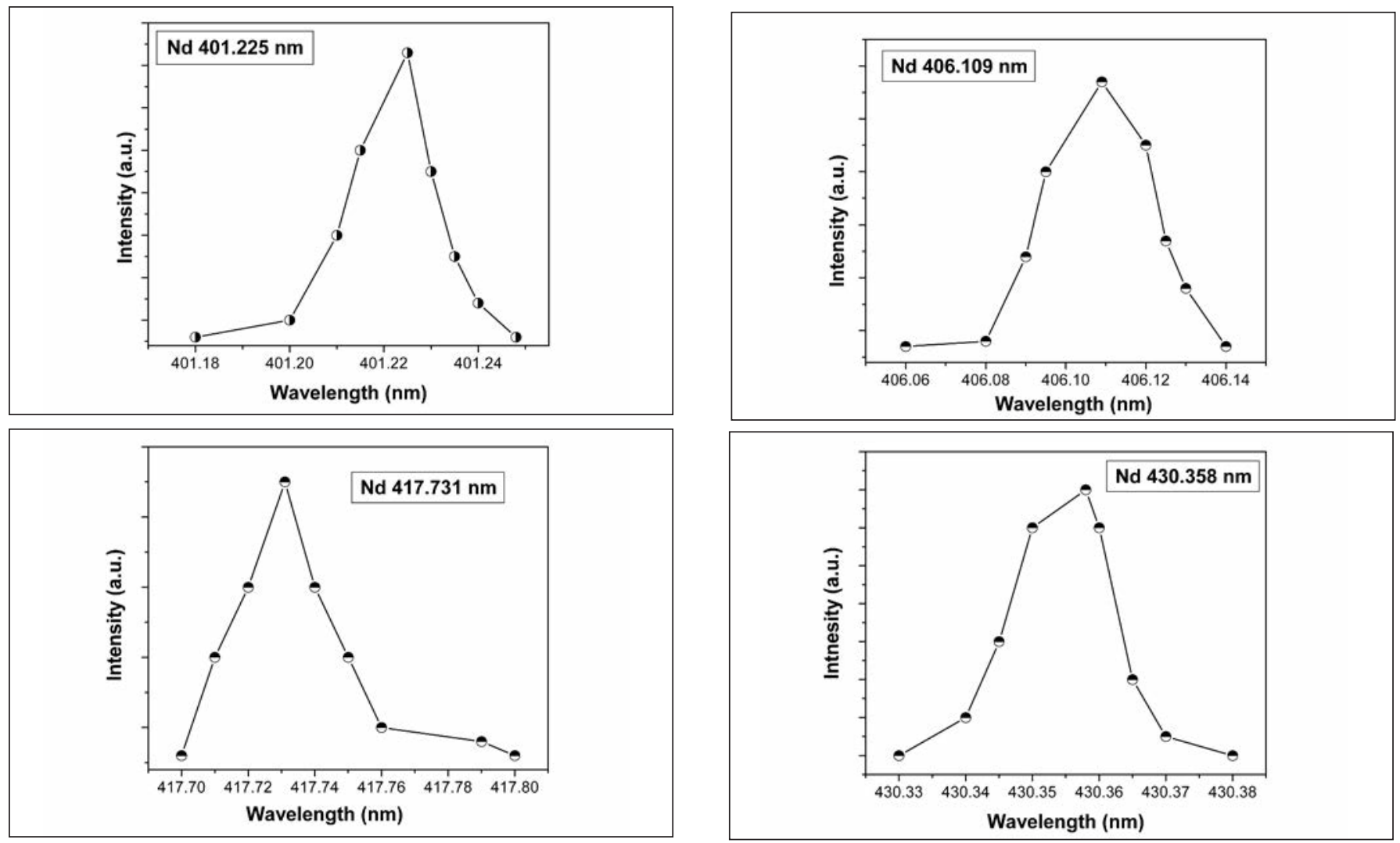

Fig. 1. Emission profiles of different analytical lines of Nd: (a) $401.225 \mathrm{~nm}$, (b) $406.109 \mathrm{~nm}$, (c) $417.731 \mathrm{~nm}$, and (d) $430.358 \mathrm{~nm}$.

\section{RESULTS AND DISCUSSION}

\section{Determination of Neodymium by ICP-AES}

For evaluating the contribution of Nd to different analytes, it is required that the correct amount be estimated in the sample. The correction factor which quantifies the contribution of $\mathrm{Nd}$ also depends on the concentration of $\mathrm{Nd}$. In this study, an ICP-AES method was developed for the direct determination of $\mathrm{Nd}$ along with different analytes including some rare earths. Based on the analytical performance, four lines of Nd were selected: $401.225 \mathrm{~nm}$, $406.109 \mathrm{~nm}, 417.731 \mathrm{~nm}$, and $430.358 \mathrm{~nm}$ (see Figure $1 \mathrm{a}-\mathrm{d}$ ). The calibration curves for these lines were established as shown in Figure 2 . The detection limit of these four $\mathrm{Nd}$ lines followed the trend:

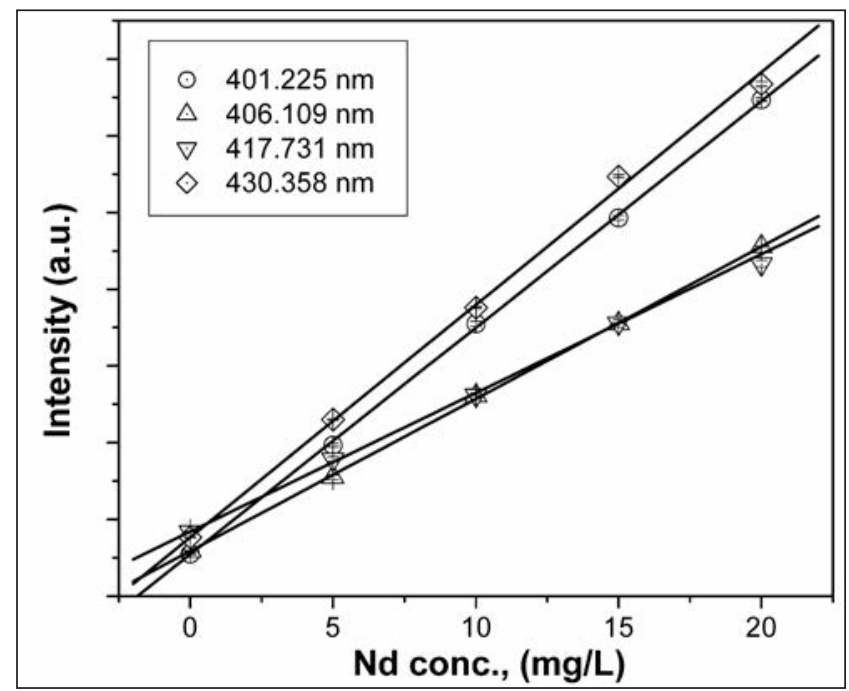

Fig. 2. Calibration curves for different analytical lines of $N d$.
$401.225 \mathrm{~nm}<406.109 \mathrm{~nm}<$ $430.358 \mathrm{~nm}<417.731 \mathrm{~nm}$, while for sensitivity it followed the trend: $401.225 \mathrm{~nm}>430.358 \mathrm{~nm}>$ $406.109 \mathrm{~nm}>417.731 \mathrm{~nm}$. The slope of the calibration curves was considered as the sensitivity of the analytical lines, whereas the detection limits were calculated as the concentration equivalent to the intensity of the blank $(\langle\mathrm{x}\rangle)+5$ times the relative standard deviation for the blank $(5 \sigma)$. Synthetic samples containing $\mathrm{Nd}$ in the con- 


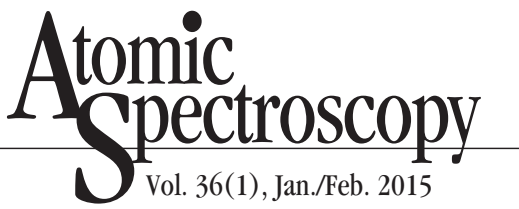

centration range $0.05-1000 \mathrm{mg} / \mathrm{L}$ were analyzed using these analytical lines. The results shown in Table II revealed that all lines of $\mathrm{Nd}$ can be suitably used for the determination of Nd up to $1000 \mathrm{mg} / \mathrm{L}$. Beyond $1000 \mathrm{mg} / \mathrm{L}$, the experiment was not carried out because of the choking problem of the nebulizer system.

\section{Spectral Interference of $\mathrm{Nd}$ on Other Analytes}

$\mathrm{Nd}$ (ground state electronic configuration $[\mathrm{Xe}] 4 \mathrm{f}^{4}, 5 \mathrm{~d}^{0}, 6 \mathrm{~s}^{2}$, and ${ }^{5} I_{4}$ ), like other members of the lanthanide series, has emission linerich spectra due to its multi-electronic nature and hence, may interfere in the analysis of other analytes most of which do not possess emission-rich spectra and, therefore, have limited lines for the analysis. The best lines for each analyte were chosen on the basis of spectral interference and analytical performance (detection limit, sensitivity, linear dynamic range, and precision).

\section{Effect on Bi, Li, Mn, and Cr}

Table III reveals the systematic spectral interference study of $\mathrm{Nd}$ on different analytical lines of $\mathrm{Bi}, \mathrm{Li}$, $\mathrm{Mn}$, and $\mathrm{Cr}$ along with their analytical performance in the presence of a Nd matrix.

\section{Bismuth (Bi)}

Among the five lines of $\mathrm{Bi}$ (306.772 nm, 190.241 nm, 206.170 $\mathrm{nm}, 222.825 \mathrm{~nm}$, and $223.061 \mathrm{~nm}$ ), the 306.772-nm line was highly sensitive and was not interfered by Nd; hence, it can be selected for the determination of $\mathrm{Bi}$ in a $\mathrm{Nd}$ matrix.

\section{Lithium (Li)}

For the 670.780-nm line of $\mathrm{Li}$, the detection limit was too low in comparison to the other three lines (274.118 nm, $323.261 \mathrm{~nm}, 460.289$ $\mathrm{nm}$ ), while the sensitivity was far too high and was the line free from interference of Nd matrix.

TABLE II

Determination of Nd by ICP-AES

\begin{tabular}{ccccc}
\hline $\begin{array}{c}\mathrm{Nd} \\
(\mathrm{mg} / \mathrm{L})\end{array}$ & $\begin{array}{c}401.225 \mathrm{~nm} \\
(\mathrm{mg} / \mathrm{L})\end{array}$ & $\begin{array}{c}430.358 \mathrm{~nm} \\
(\mathrm{mg} / \mathrm{L})\end{array}$ & $\begin{array}{c}406.109 \mathrm{~nm} \\
(\mathrm{mg} / \mathrm{L})\end{array}$ & $\begin{array}{c}417.731 \mathrm{~nm} \\
(\mathrm{mg} / \mathrm{L})\end{array}$ \\
\hline 1 & $0.98 \pm 0.03$ & $1.07 \pm 0.05$ & $0.97 \pm 0.03$ & $0.99 \pm 0.03$ \\
5 & $5.10 \pm 0.08$ & $4.94 \pm 0.07$ & $5.09 \pm 0.08$ & $5.11 \pm 0.08$ \\
10 & $10.1 \pm 0.4$ & $10.4 \pm 0.4$ & $9.5 \pm 0.6$ & $9.3 \pm 0.7$ \\
20 & $18.9 \pm 0.9$ & $20.3 \pm 0.9$ & $20.9 \pm 0.9$ & $18.2 \pm 0.9$ \\
50 & $49 \pm 2$ & $47 \pm 3$ & $53 \pm 2$ & $55 \pm 3$ \\
100 & $97 \pm 4$ & $99 \pm 5$ & $104 \pm 4$ & $103 \pm 4$ \\
500 & $503 \pm 6$ & $497 \pm 7$ & $494 \pm 6$ & $497 \pm 8$ \\
1000 & $995 \pm 9$ & $991 \pm 11$ & $995 \pm 10$ & $1010 \pm 9$ \\
\hline $\begin{array}{l}\text { D.L (mg/L). } \\
\text { Sensitivity }\end{array}$ & 0.004 & 0.057 & 0.034 & 0.06 \\
$(\mathrm{counts} / \mathrm{mg} / \mathrm{L})$ & 2961 & 2954 & 1983 & 1745 \\
Linear Dynamic & & & & \\
Range (mg/L) & $0.004-1000$ & $0.057-1000$ & $0.034-1000$ & $0.06-1000$ \\
\hline
\end{tabular}

\section{Manganese (Mn)}

The detection limit for the five lines of Mn follow the trend: $257.611 \mathrm{~nm}<403.076 \mathrm{~nm}<$ $259.373 \mathrm{~nm}<260.569 \mathrm{~nm}<$ $294.921 \mathrm{~nm}$. The 257.611-nm line was not interfered by $\mathrm{Nd}$ and showed high sensitivity; thus, it was selected for the determination of $\mathrm{Mn}$ in a Nd matrix.

\section{Chromium (Cr)}

Even though the detection limit and counts for the five lines of $\mathrm{Cr}$ (205.618 nm, 267.716 nm, 283.563 $\mathrm{nm}, 284.325 \mathrm{~nm}$, and $284.984 \mathrm{~nm}$ ) were found to be good and with reasonable \%RSD values, the $284.325 \mathrm{~nm}$ line was the only one not to be interfered by $\mathrm{Nd}$; thus, this line was selected for further studies.

Effects on Ag, B, Ba, Ca, Cd, Co, $\mathrm{Cu}, \mathrm{Fe}, \mathrm{Mg}, \mathrm{Na}, \mathrm{Sr}, \mathrm{Zn}, \mathrm{Gd}$, and Lu

All of these analytes show slight interference from Nd (see Table IV). Their analytical performance is listed in Table V. Some of the important observations from the above tables are as follows:

\section{Silver (Ag)}

The four lines selected for Ag determination were $338.289 \mathrm{~nm}$, $328.068 \mathrm{~nm}, 243.779 \mathrm{~nm}$, and $224.641 \mathrm{~nm}$. Among these, the least interfered line was 328.068 $\mathrm{nm}$. The detection limit follows the trend: $328.068 \mathrm{~nm}<338.289 \mathrm{~nm}$ $<243.779 \mathrm{~nm}<224.641 \mathrm{~nm}$, while sensitivity follows just the reverse trend. From these results it can be interpreted that the 328.068-nm line is best for the determination of Ag in a Nd matrix.

\section{Boron (B)}

The contribution from $\mathrm{Nd}$ for the four lines of $\mathrm{B}$ follows the trend: $208.959 \mathrm{~nm}<249.773 \mathrm{~nm}$ $<182.641 \mathrm{~nm}<249.677 \mathrm{~nm}$. The 208.959-nm line was found to be highly sensitive and hence, from both the spectral interference and analytical performance results, this line was best for B determination in the presence of $\mathrm{Nd}$.

\section{Barium (Ba)}

The sensitivity for the three lines of $\mathrm{Ba}$ followed the trend: 455.404 $\mathrm{nm}>230.424 \mathrm{~nm}>233.527 \mathrm{~nm}$. The 455.404-nm line was least interfered by $\mathrm{Nd}$. Therefore, this 
TABLE III

Spectral Interference of $\mathrm{Nd}$ on $\mathrm{Bi}, \mathrm{Li}, \mathrm{Mn}$ and $\mathrm{Cr}$ and the Analytical Performance of Different Analytical Lines of These Analytes

\begin{tabular}{|c|c|c|c|c|c|c|c|c|}
\hline \multirow[b]{2}{*}{ Elements } & \multirow[b]{2}{*}{$\begin{array}{l}\text { Analytical } \\
\text { Lines } \\
(\mathrm{nm})\end{array}$} & \multicolumn{3}{|c|}{ Spectral Interference from $\mathrm{Nd}$} & \multicolumn{4}{|c|}{ Analytical Performance in Presence of Nd } \\
\hline & & $\begin{array}{l}\text { Contribution } \\
\text { from } \\
1000 \mathrm{mg} / \mathrm{L} \mathrm{Nd}\end{array}$ & $\begin{array}{l}\text { Correction } \\
\text { Factor }\end{array}$ & $\begin{array}{c}\text { Tolerance } \\
\text { Level } \\
(\mathrm{mg} / \mathrm{L})\end{array}$ & $\begin{array}{c}\text { D.L. } \\
(\mathrm{mg} / \mathrm{L})\end{array}$ & $\begin{array}{l}\text { Sensitivity } \\
\text { (counts/ } \\
\mathrm{mg} / \mathrm{L}\end{array}$ & $\begin{array}{c}\text { Linear } \\
\text { Dynamic } \\
\text { Range }(\mathrm{mg} / \mathrm{L})\end{array}$ & $\%$ RSD \\
\hline \multirow[t]{5}{*}{$\mathrm{Bi}$} & 223.061 & 1.7 & $1.7 \times 10^{-3}$ & 10 & 0.3 & 346 & $0.3-900$ & 3.5 \\
\hline & 190.241 & 11.3 & $1.1 \times 10^{-2}$ & 50 & 1.3 & 39 & $1.3-600$ & 4.2 \\
\hline & 306.772 & * & - & 1000 & 0.34 & 1521 & $0.34-1000$ & 2.2 \\
\hline & 222.825 & 15.5 & $1.6 \times 10^{-2}$ & 10 & 1.5 & 48 & $1.5-500$ & 4.8 \\
\hline & 206.170 & 4.6 & $4.6 \times 10^{-3}$ & 10 & 0.44 & 76 & $0.44-800$ & 3.9 \\
\hline \multirow[t]{4}{*}{$\mathrm{Li}$} & 670.78 & $*$ & - & 1000 & 0.001 & 200525 & $0.001-1000$ & 1.0 \\
\hline & 460.289 & 5.2 & $5.2 \times 10^{-3}$ & 10 & 0.18 & 201 & 0.18-700 & 2.5 \\
\hline & 323.261 & 17.4 & $1.7 \times 10^{-2}$ & 0.05 & 0.047 & 199 & $0.047-800$ & 2.8 \\
\hline & 274.118 & 10.4 & $1.0 \times 10^{-2}$ & 10 & 0.81 & 41 & $0.81-200$ & 5.0 \\
\hline \multirow[t]{5}{*}{ Mn } & 257.611 & * & - & 1000 & 0.0016 & 12854 & $0.0016-1000$ & 1.1 \\
\hline & 259.373 & 0.1 & $1.0 \times 10^{-4}$ & 500 & 0.0037 & 10246 & $0.0037-1000$ & 1.3 \\
\hline & 260.569 & 0.17 & $1.7 \times 10^{-4}$ & 200 & 0.01 & 5858 & $0.01-950$ & 1.5 \\
\hline & 294.921 & 2.1 & $2.1 \times 10^{-3}$ & 10 & 0.042 & 1832 & $0.042-900$ & 2.2 \\
\hline & 403.076 & 2.1 & $2.1 \times 10^{-3}$ & 10 & 0.002 & 9246 & $0.002-1000$ & 1.4 \\
\hline \multirow[t]{5}{*}{$\mathrm{Cr}$} & 267.716 & 0.57 & $5.7 \times 10^{-4}$ & 10 & 0.069 & 660 & $0.069-800$ & 3.3 \\
\hline & 205.618 & 0.092 & $9.2 \times 10^{-5}$ & 500 & 0.0053 & 4588 & $0.0053-1000$ & 2.2 \\
\hline & 283.563 & 1.4 & $1.4 \times 10^{-3}$ & 10 & 0.01 & 3603 & $0.01-1000$ & 2.6 \\
\hline & 284.325 & $*$ & - & 1000 & 0.079 & 1559 & $0.079-1000$ & 3.3 \\
\hline & 284.984 & 1 & $1.0 \times 10^{-3}$ & 5 & 0.018 & 512 & $0.018-750$ & 4.1 \\
\hline
\end{tabular}

*refers no interference.

line was chosen for Ba determination in the presence of $\mathrm{Nd}$.

\section{Calcium (Ca)}

Six lines were selected for the determination of $\mathrm{Ca}: 183.801 \mathrm{~nm}$, $315.887 \mathrm{~nm}, 317.933 \mathrm{~nm}, 393.366$ $\mathrm{nm}, 396.847 \mathrm{~nm}$, and $422.673 \mathrm{~nm}$. The detection limit followed the trend: $393.366 \mathrm{~nm}<396.847 \mathrm{~nm}$ $<422.673 \mathrm{~nm}<317.933 \mathrm{~nm}<$ $315.887 \mathrm{~nm}<183.801 \mathrm{~nm}$, while the sensitivity followed just the reverse trend. The contribution by $\mathrm{Nd}$ for the 393.366-nm line was found to be less than the others and hence, it was chosen best for further study of $\mathrm{Ca}$ in a $\mathrm{Nd}$ matrix.

\section{Cadmium (Cd)}

The contribution by $\mathrm{Nd}$ for the four lines of Cd followed the trend: $226.502 \mathrm{~nm}<214.438 \mathrm{~nm}<$ $228.802 \mathrm{~nm}<361.051 \mathrm{~nm}$. The detection limit for the 226.502-nm line was lower and the sensitivity was higher than for the other lines. Thus, it was chosen for Cd determination in the presence of $\mathrm{Nd}$.

\section{Cobalt (Co)}

The detection limit and contribution of $\mathrm{Nd}$ for the four lines of $\mathrm{Co}$ were found to follow the trend: $238.892 \mathrm{~nm}<237.862<228.616$ $\mathrm{nm}<230.786$, respectively. The sensitivity for these were found to be just reversed, thus the spectral interference and analytical performance favors the Co $238.892 \mathrm{~nm}$ line for its analysis in the $\mathrm{Nd}$ matrix.

\section{Copper (Cu)}

The detection limit trend for the five lines of $\mathrm{Cu}$ was: $324.754 \mathrm{~nm}<$ $327.396 \mathrm{~nm}<224.7 \mathrm{~nm}<219.958$ $\mathrm{nm}<219.226 \mathrm{~nm}$, respectively. The $324.754 \mathrm{~nm}$ line was found to be highly sensitive and least interfered in the presence of $\mathrm{Nd}$ and thus was chosen as the best line for $\mathrm{Cu}$ in a $\mathrm{Nd}$ matrix.

\section{Iron (Fe)}

Though the Fe $239.562 \mathrm{~nm}$ line (out of ten lines: $259.941 \mathrm{~nm}$, $238.204 \mathrm{~nm}, 239.562 \mathrm{~nm}, 244.451$ $\mathrm{nm}, 261.187 \mathrm{~nm}, 262.567 \mathrm{~nm}$, $262.829 \mathrm{~nm}, 275.573 \mathrm{~nm}, 241.331$ $\mathrm{nm}$, and $373.486 \mathrm{~nm}$ ) was not the best but showed acceptable analytical performance, it was also least interfered by Nd. Hence, it is considered best for Fe determination in the presence of $\mathrm{Nd}$. 


\section{Atomic Apectroscopy \\ $\bigcirc$ Vol. 36(1), Jan./Feb. 2015}

TABLE IV

Spectral Interference of $\mathrm{Nd}$ on $\mathrm{Ag}, \mathrm{B}, \mathrm{Ba}, \mathrm{Ca}, \mathrm{Cd}, \mathrm{Co}, \mathrm{Cu}, \mathrm{Fe}, \mathrm{Mg}, \mathrm{Na}, \mathrm{Sr}, \mathrm{Zn}, \mathrm{Gd}$, and Lu Analytes

\begin{tabular}{|c|c|c|c|c|c|c|c|c|c|}
\hline Elements & $\begin{array}{l}\text { Analytical } \\
\text { Lines } \\
(\mathrm{nm})\end{array}$ & $\begin{array}{l}\text { Contribution } \\
\text { from } 1000 \\
\mathrm{mg} / \mathrm{L} \text { of } \mathrm{Nd}\end{array}$ & $\begin{array}{l}\text { Correction } \\
\text { Factor }\end{array}$ & $\begin{array}{c}\text { Tolerance } \\
\text { Level } \\
(\mathrm{mg} / \mathrm{L})\end{array}$ & Elements & $\begin{array}{l}\text { Analytical } \\
\text { Lines } \\
\text { (nm) }\end{array}$ & $\begin{array}{l}\text { Contribution } \\
\text { from } 1000 \\
\mathrm{mg} / \mathrm{L} \text { of } \mathrm{Nd}\end{array}$ & $\begin{array}{c}\text { Correction } \\
\text { Factor }\end{array}$ & $\begin{array}{c}\begin{array}{c}\text { Tolerance } \\
\text { Level } \\
(\mathrm{mg} / \mathrm{L})\end{array} \\
\end{array}$ \\
\hline \multirow[t]{4}{*}{ Ag } & 338.289 & 1.5 & $1.5 \times 10^{-3}$ & 10 & \multirow[t]{8}{*}{$\mathrm{Fe}$} & 259.941 & 1.3 & $1.3 \times 10^{-3}$ & 10 \\
\hline & 328.068 & 0.34 & $3.4 \times 10^{-4}$ & 50 & & 238.204 & 0.81 & $8.1 \times 10^{-4}$ & 10 \\
\hline & 224.641 & 20.3 & $2.0 \times 10^{-2}$ & 10 & & 239.562 & 0.31 & $3.1 \times 10^{-4}$ & 50 \\
\hline & 243.779 & 2.1 & $2.1 \times 10^{-3}$ & 10 & & 244.451 & 4.4 & $4.4 \times 10^{-3}$ & 10 \\
\hline \multirow[t]{4}{*}{ B } & 249.773 & 0.31 & $3.1 \times 10^{-4}$ & 50 & & 261.187 & 0.95 & $9.5 \times 10^{-4}$ & 10 \\
\hline & 249.677 & 0.52 & $5.2 \times 10^{-4}$ & 10 & & 262.567 & 10.4 & $1.0 \times 10^{-2}$ & 10 \\
\hline & 182.641 & 0.46 & $4.6 \times 10^{-4}$ & 50 & & 262.829 & 2.6 & $2.6 \times 10^{-3}$ & 10 \\
\hline & 208.959 & 0.10 & $1.0 \times 10^{-4}$ & 500 & & 275.573 & 1.9 & $1.9 \times 10^{-3}$ & 10 \\
\hline \multirow[t]{6}{*}{$\mathrm{Ca}$} & 396.847 & 0.98 & $9.8 \times 10^{-4}$ & 10 & & 241.331 & 3.9 & $3.9 \times 10^{-3}$ & 10 \\
\hline & 393.366 & 0.32 & $3.2 \times 10^{-4}$ & 200 & & 373.486 & \# & - & - \\
\hline & 317.933 & 0.96 & $9.6 \times 10^{-4}$ & 10 & $\mathrm{Na}$ & 589.592 & 0.24 & $2.4 \times 10^{-4}$ & 200 \\
\hline & 315.887 & 2.0 & $2.0 \times 10^{-3}$ & 200 & & 588.995 & 0.14 & $1.4 \times 10^{-4}$ & 500 \\
\hline & 183.801 & 10.0 & $1.0 \times 10^{-2}$ & 50 & & 330.237 & \# & - & - \\
\hline & 422.673 & 5.6 & $5.6 \times 10^{-3}$ & 10 & & 330.298 & $\#$ & - & - \\
\hline \multirow[t]{4}{*}{$\mathrm{Cd}$} & 214.438 & 0.16 & $1.6 \times 10^{-4}$ & 500 & \multirow[t]{3}{*}{$\mathrm{Sr}$} & 407.771 & 0.34 & $3.4 \times 10^{-4}$ & 200 \\
\hline & 226.502 & 0.13 & $1.3 \times 10^{-4}$ & 500 & & 421.552 & 0.09 & $9.0 \times 10^{-5}$ & 500 \\
\hline & 228.802 & 0.34 & $3.4 \times 10^{-4}$ & 10 & & 460.733 & 15.7 & $1.6 \times 10^{-2}$ & 1 \\
\hline & 361.051 & \# & - & - & \multirow[t]{4}{*}{$\mathrm{Zn}$} & 213.856 & 0.09 & $9.0 \times 10^{-5}$ & 500 \\
\hline \multirow[t]{4}{*}{ Co } & 228.616 & 0.81 & $8.1 \times 10-4$ & 10 & & 206.200 & 2.6 & $2.6 \times 10^{-3}$ & 10 \\
\hline & 238.892 & 0.33 & $3.3 \times 10^{-4}$ & 10 & & 202.613 & 0.53 & $5.3 \times 10^{-4}$ & 50 \\
\hline & 230.786 & 1.1 & $1.1 \times 10^{-3}$ & 10 & & 334.502 & \# & - & - \\
\hline & 237.862 & 0.44 & $4.4 \times 10^{-4}$ & 10 & \multirow[t]{4}{*}{ Gd } & 342.247 & 0.36 & $3.6 \times 10^{-4}$ & 50 \\
\hline \multirow[t]{5}{*}{$\mathrm{Cu}$} & 324.754 & 0.22 & $2.2 \times 10^{-4}$ & 200 & & 335.047 & 8.2 & $8.2 \times 10^{-3}$ & 5 \\
\hline & 327.396 & 2.5 & $2.5 \times 10^{-3}$ & 10 & & 336.223 & 0.69 & $6.9 \times 10^{-4}$ & 200 \\
\hline & 224.700 & 0.42 & $4.2 \times 10^{-4}$ & 50 & & 335.862 & 5.6 & $5.6 \times 10^{-3}$ & 0.05 \\
\hline & 219.226 & 1.2 & $1.2 \times 10^{-3}$ & 10 & \multirow[t]{4}{*}{$\mathrm{Lu}$} & 261.542 & 0.14 & $1.4 \times 10^{-4}$ & 500 \\
\hline & 219.958 & 0.78 & $7.8 \times 10^{-4}$ & 10 & & 291.139 & 0.24 & $2.4 \times 10^{-4}$ & 10 \\
\hline \multirow[t]{5}{*}{$\mathrm{Mg}$} & 279.553 & 0.10 & $1.0 \times 10^{-4}$ & 200 & & 307.760 & 0.63 & $6.3 \times 10^{-4}$ & 5 \\
\hline & 280.270 & 0.12 & $1.2 \times 10^{-4}$ & 200 & & 219.554 & 0.47 & $4.7 \times 10^{-4}$ & 10 \\
\hline & 285.213 & 0.14 & $1.4 \times 10^{-4}$ & 200 & \multirow[t]{3}{*}{$\mathrm{Ba}$} & 455.404 & 0.34 & $3.4 \times 10^{-4}$ & 10 \\
\hline & 279.079 & 1.3 & $1.3 \times 10^{-3}$ & 10 & & 233.527 & 1.5 & $1.5 \times 10^{-3}$ & 1 \\
\hline & 202.647 & 2.7 & $2.7 \times 10^{-3}$ & 0.05 & & 230.424 & 0.83 & $8.3 \times 10^{-4}$ & 10 \\
\hline
\end{tabular}

Note: \#significant interference and the line should therefore be rejected.

\section{Magnesium (Mg)}

The contribution of $\mathrm{Nd}$ to the five lines of $\mathrm{Mg}$ followed the trend: $279.553 \mathrm{~nm}<280.270 \mathrm{~nm}<$ $285.213 \mathrm{~nm}<279.079 \mathrm{~nm}<$ $202.647 \mathrm{~nm}$, respectively. The analytical performance result for $279.553 \mathrm{~nm}$ line of $\mathrm{Mg}$ was far better than the other lines and hence, seemed to be the best line for $\mathrm{Mg}$ determination in the $\mathrm{Nd}$ matrix.

\section{Sodium (Na)}

Of the four lines of $\mathrm{Na}, 330.237$ $\mathrm{nm}, 330.298 \mathrm{~nm}, 588.995 \mathrm{~nm}$, and $589.592 \mathrm{~nm}$, the last two lines slightly interfered, and the $\mathbf{5 8 8 . 9 9 5}$ nm line least interfered. The sensi- tivity of the lines followed the trend $588.995 \mathrm{~nm}>589.592 \mathrm{~nm}>$ $330.237 \mathrm{~nm}>330.298 \mathrm{~nm}$, respectively, where the detection limit for the $588.995 \mathrm{~nm}$ and the $\mathbf{5 8 9 . 5 9 2}$ $\mathrm{nm}$ line was almost similar. Thus, considering all factors, the $\mathbf{5 8 8 . 9 9 5}$ $\mathrm{nm}$ line seems to be the best line for $\mathrm{Na}$ in a $\mathrm{Nd}$ matrix. 
TABLE V

Analytical Performance of Ag, B, Ba, Ca, Cd, Co, Cu, Fe, Mg, Na, Sr, Zn, Gd, and Lu in the Presence of Nd

\begin{tabular}{|c|c|c|c|c|c|c|c|c|c|c|c|}
\hline $\begin{array}{l}\text { Ele } \\
\text { ments }\end{array}$ & $\begin{array}{l}\text { Analytical } \\
\text { Lines } \\
(\mathrm{nm})\end{array}$ & $\begin{array}{c}\text { D.L. } \\
(\mathrm{mg} / \mathrm{L})\end{array}$ & $\begin{array}{l}\text { Sensitivity } \\
\text { (counts/ } \\
\text { mg/L) }\end{array}$ & $\begin{array}{l}\text { Linear } \\
\text { Dynamic } \\
\text { Range } \\
(\mathrm{mg} / \mathrm{L})\end{array}$ & $\begin{array}{l}\text { (\%) } \\
\text { RSD }\end{array}$ & $\begin{array}{l}\text { Ele- } \\
\text { ments }\end{array}$ & $\begin{array}{l}\text { Analytical } \\
\text { Lines } \\
(\mathrm{nm})\end{array}$ & $\begin{array}{c}\text { D.L. } \\
\text { (mg/L) }\end{array}$ & $\begin{array}{c}\text { Sensitivity } \\
\text { (counts/ } \\
\text { mg/L) }\end{array}$ & $\begin{array}{l}\text { Linear } \\
\text { Dynamic } \\
\text { Range } \\
(\mathrm{mg} / \mathrm{L})\end{array}$ & $\begin{array}{l}\text { (\%) } \\
\text { RSD }\end{array}$ \\
\hline \multirow[t]{4}{*}{$\mathrm{Ag}$} & 224.641 & 1.6 & 38.6 & $1.6-800$ & 5.0 & \multirow[t]{10}{*}{$\mathrm{Fe}$} & 238.204 & 0.093 & 800 & $0.093-900$ & 4.2 \\
\hline & 243.779 & 0.22 & 346 & $0.22-700$ & 5.0 & & 239.562 & 0.032 & 2063 & $0.032-1000$ & 2.2 \\
\hline & 328.068 & 0.012 & 13737 & $0.012-1000$ & 2.1 & & 241.331 & 0.27 & 229 & $0.27-600$ & 4.5 \\
\hline & 338.289 & 0.018 & 7156 & $0.018-1000$ & 2.0 & & 244.451 & 0.53 & 157 & $0.53-900$ & 5.0 \\
\hline \multirow[t]{4}{*}{ B } & 182.641 & 0.024 & 757 & $0.024-800$ & 4.9 & & 259.941 & 0.018 & 1689 & $0.0018-1000$ & 3.2 \\
\hline & 208.959 & 0.014 & 4309 & $0.014-1000$ & 1.8 & & 261.187 & 0.025 & 943 & $0.025-970$ & 4.1 \\
\hline & 249.677 & 0.025 & 1013 & $0.025-1000$ & 2.3 & & 262.567 & 0.29 & 136 & $0.29-750$ & 4.9 \\
\hline & 249.773 & 0.014 & 1587 & $0.014-1000$ & 2.1 & & 262.829 & 0.38 & 194 & $0.38-1000$ & 4.8 \\
\hline \multirow[t]{6}{*}{$\mathrm{Ca}$} & 183.801 & 0.53 & 29.6 & $0.53-900$ & 5.0 & & 275.573 & 0.14 & 293 & $0.14-900$ & 4.5 \\
\hline & 315.887 & 0.10 & 1114 & $0.10-800$ & 4.3 & & 373.486 & 0.052 & 2173 & $0.052-1000$ & 2.1 \\
\hline & 317.933 & 0.039 & 3613 & $0.039-950$ & 3.1 & \multirow[t]{4}{*}{$\mathrm{Na}$} & 330.237 & 0.81 & 84.2 & $0.81-900$ & 3.7 \\
\hline & 393.366 & 0.0013 & 521209 & $0.0013-1000$ & 1.1 & & 330.298 & 1.7 & 52.1 & $1.7-950$ & 5.0 \\
\hline & 396.847 & 0.0017 & 400267 & $0.0017-1000$ & 1.1 & & 588.995 & 0.016 & 53419 & $0.016-1000$ & 1.3 \\
\hline & 422.673 & 0.0041 & 25334 & $0.0041-1000$ & 1.4 & & 589.592 & 0.01 & 32488 & $0.01-1000$ & 1.5 \\
\hline \multirow[t]{4}{*}{$\mathrm{Cd}$} & 214.438 & 0.0063 & 5244 & $0.0063-1000$ & 2.3 & \multirow[t]{3}{*}{$\mathrm{Sr}$} & 407.771 & 0.0002 & 346709 & $0.0002-1000$ & 0.8 \\
\hline & 226.502 & 0.0048 & 11494 & $0.0048-1000$ & 1.8 & & 421.520 & 0.0006 & 262155 & $0.0006-1000$ & 0.9 \\
\hline & 228.802 & 0.025 & 1884 & $0.025-800$ & $2 . .9$ & & 460.733 & 0.033 & 2307 & $0.033-1000$ & 1.6 \\
\hline & 361.051 & 0.17 & 318 & $0.17-500$ & 3.7 & \multirow[t]{4}{*}{$\mathrm{Zn}$} & 202.613 & 0.008 & 2058 & $0.008-1000$ & 2.2 \\
\hline \multirow[t]{4}{*}{$\mathrm{Co}$} & 228.616 & 0.049 & 826 & $0.049-800$ & 4.0 & & 206.200 & 0.21 & 157 & $0.21-500$ & 3.9 \\
\hline & 230.786 & 0.14 & 658 & $0.14-900$ & 4.6 & & 213.856 & 0.001 & 18061 & $0.001-1000$ & 2.0 \\
\hline & 237.862 & 0.043 & 1457 & $0.043-1000$ & 3.9 & & 334.502 & 0.12 & 453 & $0.12-950$ & 3.5 \\
\hline & 238.892 & 0.033 & 2001 & $0.033-1000$ & 3.6 & \multirow[t]{4}{*}{ Gd } & 335.047 & 0.007 & 6474 & $0.007-1000$ & 2.3 \\
\hline \multirow[t]{5}{*}{$\mathrm{Cu}$} & 219.226 & 0.12 & 377 & $0.12-750$ & 3.2 & & 335.862 & 0.015 & 3729 & $0.015-1000$ & 3.4 \\
\hline & 219.958 & 0.04 & 762 & $0.04-900$ & 2.8 & & 336.223 & 0.027 & 4423 & $0.027-1000$ & 3.0 \\
\hline & 224.700 & 0.038 & 1859 & $0.038-750$ & 2.3 & & 342.247 & 0.014 & 5073 & $0.014-1000$ & 2.4 \\
\hline & 324.754 & 0.0026 & 52030 & $0.0026-1000$ & 1.0 & \multirow[t]{4}{*}{ Lu } & 219.554 & 0.045 & 911 & $0.045-800$ & 4.3 \\
\hline & 327.396 & 0.0034 & 34031 & $0.0034-1000$ & 1.1 & & 261.542 & 0.003 & 7454 & $0.003-1000$ & 1.2 \\
\hline \multirow[t]{5}{*}{$\mathrm{Mg}$} & 202.647 & 0.026 & 496 & $0.026-800$ & 2.4 & & 291.139 & 0.021 & 2427 & $0.021-1000$ & 2.8 \\
\hline & 279.079 & 0.23 & 390 & $0.23-900$ & 3.4 & & 307.760 & 0.095 & 2856 & $0.095-1000$ & 2.7 \\
\hline & 279.553 & 0.0005 & 653523 & $0.0005-1000$ & 0.85 & \multirow[t]{3}{*}{$\mathrm{Ba}$} & 230.424 & 0.012 & 793 & $0.012-950$ & 3.8 \\
\hline & 280.270 & 0.0009 & 48536 & $0.0009-1000$ & 0.94 & & 233.527 & 0.091 & 388 & $0.091-950$ & 4.8 \\
\hline & 285.213 & 0.0046 & 15455 & $0.0046-1000$ & 1.6 & & 455.404 & 0.028 & 2479 & $0.028-1000$ & 1.6 \\
\hline
\end{tabular}

\section{Strontium (Sr)}

The three lines chosen for the determination of Sr were 407.771 $\mathrm{nm}, 421.552 \mathrm{~nm}$, and $460.733 \mathrm{~nm}$. The $421.552 \mathrm{~nm}$ line interfered less and possesses good analytical performance; thus, the $421.552 \mathrm{~nm}$ line of $\mathrm{Sr}$ seems to be appropriate for its determination in a Nd matrix.

\section{Zinc (Zn)}

The detection limit trend for the four lines of $\mathrm{Zn}$ was: $213.856 \mathrm{~nm}$ $<202.613 \mathrm{~nm}<334.502 \mathrm{~nm}<$ $206.200 \mathrm{~nm}$, respectively. The $213.856 \mathrm{~nm}$ line was found to be much less interfered, has the highest sensitivity and lowest detection limit. Thus, the $213.856 \mathrm{~nm}$ line was the best choice for $\mathrm{Zn}$ determination in presence of $\mathrm{Nd}$.

\section{Gadolinium (Gd)}

The analytical performance of each of the four lines of $\mathrm{Gd}$ $(335.047 \mathrm{~nm}, 335.862 \mathrm{~nm}, 336.223$ $\mathrm{nm}$, and $342.247 \mathrm{~nm}$ ) seems to be close to each other with a reason- 


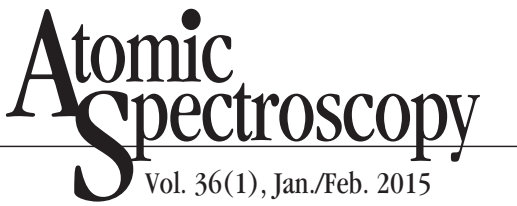

TABLE VI

Spectral Interference of Nd on Ga, Ni, Al, Tl, and Dy and the Analytical Performance of Different Analytical Lines of These Analytes

\begin{tabular}{|c|c|c|c|c|c|c|c|c|}
\hline \multirow[b]{2}{*}{ Elements } & \multirow[b]{2}{*}{$\begin{array}{l}\text { Analytical } \\
\text { Lines } \\
\text { (nm) }\end{array}$} & \multicolumn{3}{|c|}{ Spectral Interference from $\mathrm{Nd}$} & \multicolumn{4}{|c|}{ Analytical Performance in Presence of $\mathrm{Nd}$} \\
\hline & & $\begin{array}{l}\text { Contribution } \\
\text { from } \\
1000 \mathrm{mg} / \mathrm{L} \mathrm{Nd}\end{array}$ & $\begin{array}{l}\text { Correction } \\
\text { Factor }\end{array}$ & $\begin{array}{l}\text { Tolerance } \\
\text { Level } \\
(\mathrm{mg} / \mathrm{L})\end{array}$ & $\begin{array}{c}\text { D.L. } \\
(\mathrm{mg} / \mathrm{L})\end{array}$ & $\begin{array}{l}\text { Sensitivity } \\
\text { (counts/ } \\
\mathrm{mg} / \mathrm{L}\end{array}$ & $\begin{array}{c}\text { Linear } \\
\text { Dynamic } \\
\text { Range }(\mathrm{mg} / \mathrm{L})\end{array}$ & $\%$ RSD \\
\hline \multirow[t]{3}{*}{ Ga } & 417.206 & 0.76 & $7.6 \times 10_{-4}$ & 10 & 0.03 & 7693 & $0.03-1000$ & 1.1 \\
\hline & 294.364 & 5.3 & $5.3 \times 10^{-3}$ & 0 & 0.03 & 864 & $0.03-1000$ & 3.3 \\
\hline & 287.424 & 11.6 & $1.2 \times 10^{-2}$ & 0 & 0.26 & 182 & $0.26-800$ & 4.2 \\
\hline \multirow[t]{6}{*}{$\mathrm{Ni}$} & 231.604 & 0.69 & $6.9 \times 10^{-4}$ & 10 & 0.01 & 853 & $0.01-850$ & 3.6 \\
\hline & 221.648 & 0.55 & $5.5 \times 10^{-4}$ & 5 & 0.13 & 848 & $0.13-900$ & 3.2 \\
\hline & 232.003 & 1.5 & $1.5 \times 10^{-3}$ & 10 & 0.07 & 704 & $0.07-800$ & 3.6 \\
\hline & 227.021 & 1.4 & $1.4 \times 10^{-3}$ & 10 & 0.05 & 154 & $0.05-650$ & 4.6 \\
\hline & 300.249 & 5.5 & $5.5 \times 10^{-3}$ & 10 & 0.11 & 642 & $0.11-900$ & 3.9 \\
\hline & 341.476 & 2 & $2.0 \times 10^{-3}$ & 10 & 0.01 & 4201 & $0.01-1000$ & 1.7 \\
\hline \multirow[t]{4}{*}{ Al } & 396.152 & 4.8 & $4.8 \times 10^{-3}$ & 10 & 0.01 & 10037 & $0.01-1000$ & 1.0 \\
\hline & 394.401 & 9.6 & $9.6 \times 10^{-3}$ & 0 & 0.02 & 3109 & $0.02-1000$ & 1.6 \\
\hline & 308.215 & 0.6 & $6.0 \times 10^{-4}$ & 10 & 0.04 & 1822 & $0.04-1000$ & 1.5 \\
\hline & 309.271 & * & - & 1000 & 1.9 & 3130 & $1.9-900$ & 1.0 \\
\hline \multirow[t]{2}{*}{$\mathrm{Tl}$} & 190.864 & 0.69 & $6.9 \times 10^{-4}$ & 10 & 0.03 & 848 & $0.03-1000$ & 2.1 \\
\hline & 276.787 & \# & - & - & 0.83 & 27.2 & $0.83-600$ & 5.0 \\
\hline \multirow[t]{5}{*}{ Dy } & 353.170 & 4 & $4.0 \times 10^{-3}$ & 10 & 0.02 & 14075 & $0.02-1000$ & 1.0 \\
\hline & 394.468 & 5.1 & $5.1 \times 10^{-3}$ & 10 & 0.04 & 3884 & $0.04-1000$ & 2.2 \\
\hline & 340.780 & 0.44 & $4.4 \times 10^{-4}$ & 10 & 0.02 & 4300 & $0.02-1000$ & 1.8 \\
\hline & 353.602 & 8.1 & $8.1 \times 10^{-3}$ & 10 & 0.05 & 4040 & $0.05-1000$ & 2.1 \\
\hline & 364.540 & 2.8 & $2.8 \times 10^{-3}$ & 10 & 0.03 & 4864 & $0.03-1000$ & 2.6 \\
\hline
\end{tabular}

Note: *No interference, \#Significant Interference.

able \% RSD value. However, the $342.247 \mathrm{~nm}$ line was found to be least interfered by $\mathrm{Nd}$ and hence, was selected for the estimation of $\mathrm{Gd}$ in the presence of $\mathrm{Nd}$.

\section{Lutetium (Lu)}

The contribution of $\mathrm{Nd}$ and the detection limit for the four lines of Lu followed the trend: $261.542 \mathrm{~nm}$ $<291.139 \mathrm{~nm}<219.554 \mathrm{~nm}<$ $307.760 \mathrm{~nm}$. Since the $261.542 \mathrm{~nm}$ line is more sensitive than the other lines, it was considered the best line for Lu estimation in a $\mathrm{Nd}$ matrix.

\section{Effects on Ga, Ni, Al, T1, and Dy}

These analytes showed a slightly higher interference from $\mathrm{Nd}$ than the previously mentioned analytes. The results are listed in Table VI including the analytical performance.

\section{Gallium (Ga)}

Three lines (287.424 nm, $294.364 \mathrm{~nm}$, and $417.206 \mathrm{~nm}$ ) were chosen for $\mathrm{Ga}$. It was found that the $294.364 \mathrm{~nm}$ and $417.206 \mathrm{~nm}$ lines have similar detection limits with reasonable \%RSD values, and are definitely better than the $287.424 \mathrm{~nm}$ line. Since the $\mathbf{4 1 7 . 2 0 6}$ nm line interfered least and has a tolerance level of $10 \mathrm{mg} / \mathrm{L}$, it was considered the best line for Ga determination in a $\mathrm{Nd}$ matrix.

\section{Nickel (Ni)}

In the case of $\mathrm{Ni}$, of the six lines (221.648 nm, $227.021 \mathrm{~nm}, 231.604$ $\mathrm{nm}, 232.003 \mathrm{~nm}, 300.249 \mathrm{~nm}$, and $341.476 \mathrm{~nm}$ ), only the $221.648 \mathrm{~nm}$ and $231.604 \mathrm{~nm}$ lines have a closer and lesser value of contribution due to $\mathrm{Nd}$. However, the detection limit of the $231.604 \mathrm{~nm}$ line was less and with a good \%RSD value than with the $21.648 \mathrm{~nm}$ line. Thus, the $231.604 \mathrm{~nm}$ line, with a tolerance level of $10 \mathrm{mg} / \mathrm{L}$, seems to be the best choice for analyzing $\mathrm{Ni}$ in the presence of $\mathrm{Nd}$. 


\section{Aluminum (Al)}

For $\mathrm{Al}$, the $309.271 \mathrm{~nm}$ line (out of the four chosen lines of 308.215 $\mathrm{nm}, 309.271 \mathrm{~nm}, 394.401 \mathrm{~nm}$, and $396.152 \mathrm{~nm}$ ) was found not to be interfered by $\mathrm{Nd}$. The detection limit of this line was very high, had a poor \% RSD value, and was thus not considered. Since the 308.215 $\mathrm{nm}$ line was least interfered and has reasonable analytical performance, it was selected as the best line for $\mathrm{Al}$ in the presence of $\mathrm{Nd}$.

\section{Tellurium (T1)}

For $\mathrm{Tl}$, the $190.864 \mathrm{~nm}$ and $276.787 \mathrm{~nm}$ lines were chosen. The analytical performance for the $190.864 \mathrm{~nm}$ line was superior over the $276.787 \mathrm{~nm}$ line, also has less contribution of $\mathrm{Nd}$, and was therefore chosen to estimate Tl up to $10 \mathrm{mg} / \mathrm{L}$ concentration of $\mathrm{Nd}$.

\section{Dysprosium (Dy)}

The five lines of Dy (340.780 $\mathrm{nm}, 353.170 \mathrm{~nm}, 353.602 \mathrm{~nm}$, $364.540 \mathrm{~nm}$, and $394.468 \mathrm{~nm}$ ) have good detection limit with an appropriate $\%$ RSD value. The contribution by $\mathrm{Nd}$ followed the trend: $340.780 \mathrm{~nm}<364.540 \mathrm{~nm}$ $<353.170 \mathrm{~nm}<394.468 \mathrm{~nm}<$ $353.602 \mathrm{~nm}$. Therefore, the $340.780 \mathrm{~nm}$ line, with a tolerance level of $10 \mathrm{mg} / \mathrm{L}$, is considered the best line for Dy determination in the presence of $\mathrm{Nd}$.

\section{Effects on Pb, In, Ce, La, and Pr}

The analytes listed in Table VII show their spectral interference and analytical performance which cause a higher interference in the presence of a Nd matrix than the previously discussed analytes.

\section{Lead (Pb)}

Four lines of $\mathrm{Pb}(220.353 \mathrm{~nm}$ $261.418 \mathrm{~nm}, 283.305 \mathrm{~nm}$, and $405.778 \mathrm{~nm}$ ) were chosen for this study. The sensitivity of the $283.305 \mathrm{~nm}$ and $405.778 \mathrm{~nm}$ lines was found to be similar to each other and better than the remaining two lines due to their low detection limit and less interference from $\mathrm{Nd}$. Thus, the $283.305 \mathrm{~nm}$ line, with a tolerance level of $10 \mathrm{mg} / \mathrm{L}$, was chosen as the best line for $\mathrm{Pb}$ determination in the presence of $\mathrm{Nd}$.

\section{Indium (In)}

The detection limit trend for the four lines of In was found to be as follows: $230.606 \mathrm{~nm}<$ $325.609 \mathrm{~nm}<303.936 \mathrm{~nm}<$ $451.131 \mathrm{~nm}$. The $230.606 \mathrm{~nm}$ line, with a tolerance level of $10 \mathrm{mg} / \mathrm{L}$, was found to be least interfered and was, therefore, chosen as the best line for In determination in a $\mathrm{Nd}$ matrix.

\section{Cesium (Cs)}

Among the five lines of Cs (393.373 nm, $413.380 \mathrm{~nm}, 413.765$ $\mathrm{nm}, 418.660 \mathrm{~nm}$, and $448.691 \mathrm{~nm})$, the $413.380 \mathrm{~nm}$ line, with a tolerance level of $1 \mathrm{mg} / \mathrm{L}$, and the $413.765 \mathrm{~nm}$ line, with a tolerance level of $10 \mathrm{mg} / \mathrm{L}$, showed peculiar results. The 413.380 line has a better detection limit but a poor spectral interference, while the 413.765 nm line showed a completely reverse result. These two lines were found superior over the other three lines and thus, both of them were chosen as the best line for Cs determination in the presence of Nd.

\section{Lanthanum (La)}

The detection limit for the three lines of La (333.749 nm, 379.478 $\mathrm{nm}$, and $408.672 \mathrm{~nm}$ ) were found to be reasonable with an appropriate $\%$ RSD value. However, the contribution of $\mathrm{Nd}$ for these three lines followed the trend: $333.749 \mathrm{~nm}<$ $379.478 \mathrm{~nm}<408.672 \mathrm{~nm}$, respectively. Thus, the $333.749 \mathrm{~nm}$ line, with a tolerance level of $0.05 \mathrm{mg} / \mathrm{L}$, can be considered the best line for La determination in the presence of $\mathrm{Nd}$.

\section{Praeseodymium (Pr)}

The analytical performance results for the four lines of $\mathrm{Pr}$ (411.846 nm, $414.311 \mathrm{~nm}, 417.939$ $\mathrm{nm}$, and $422.535 \mathrm{~nm}$ ) were found to be very close to each other. However, the contribution of $\mathrm{Nd}$ is least for the $422.535 \mathrm{~nm}$ line, with a tolerance level of $10 \mathrm{mg} / \mathrm{L}$, and was chosen as the best line for Pr determination in a Nd matrix.

\section{Determination of Analytes at Trace Levels in a Nd Matrix Without Chemical Separation Using Synthetic Samples}

All of the analytes were divided into four groups according to their behavior in a Nd matrix and eight synthetic samples were prepared using standard reference material of the individual elemental solutions procured from E. Merck, Germany. The synthetic samples were fed into the plasma without any chemical separation and the analytes were determined using the most suitable analytical lines.

\section{Composition of the Synthetic samples}

- Synthetic sample 1 (S1): $0.1 \mathrm{mg} / \mathrm{L}$ of $\mathrm{Bi}, \mathrm{Li}, \mathrm{Mn}, \mathrm{Cr}$ in 1000 $\mathrm{mg} / \mathrm{L}$ of $\mathrm{Nd}$

- Synthetic sample 2 (S2): $1 \mathrm{mg} / \mathrm{L}$ of $\mathrm{Bi}, \mathrm{Li}, \mathrm{Mn}, \mathrm{Cr}$ in 1000 $\mathrm{mg} / \mathrm{L}$ of $\mathrm{Nd}$

- Synthetic sample 3 (S3): $0.6 \mathrm{mg} / \mathrm{L}$ of $\mathrm{Ga}, \mathrm{Ni}, \mathrm{Al}, \mathrm{Tl}$ and Dy in $1000 \mathrm{mg} / \mathrm{L}$ of $\mathrm{Nd}$

- Synthetic sample 4 (S4): $2 \mathrm{mg} / \mathrm{L}$ of $\mathrm{Ga}, \mathrm{Ni}, \mathrm{Al}, \mathrm{Tl}$ and Dy in $1000 \mathrm{mg} / \mathrm{L}$ of $\mathrm{Nd}$

- Synthetic sample 5 (S5): $2 \mathrm{mg} / \mathrm{L}$ of $\mathrm{Pb}, \mathrm{In}, \mathrm{Pr}$ and $5 \mathrm{mg} / \mathrm{L}$ of $\mathrm{Cs}, \mathrm{La}$ in $1000 \mathrm{mg} / \mathrm{L}$ of $\mathrm{Nd}$

- Synthetic sample 6 (S6): $5 \mathrm{mg} / \mathrm{L}$ of $\mathrm{Pb}$, In, $\mathrm{Pr}$ and $10 \mathrm{mg} / \mathrm{L}$ of $\mathrm{Cs}, \mathrm{La}$ in $1000 \mathrm{mg} / \mathrm{L}$ of $\mathrm{Nd}$ 


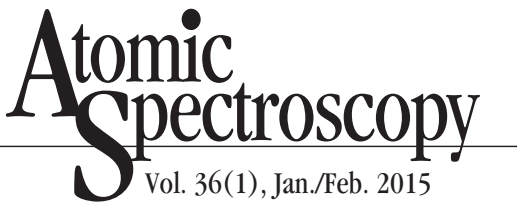

TABLE VII

Spectral Interference of $\mathrm{Nd}$ on $\mathrm{Pb}, \mathrm{Ce}, \mathrm{In}, \mathrm{La}$ and $\mathrm{Pr}$ Analytes and the Analytical Performance of Different Analytical Lines of These Analytes

\begin{tabular}{|c|c|c|c|c|c|c|c|c|}
\hline \multirow[b]{2}{*}{ Elements } & \multirow[b]{2}{*}{$\begin{array}{l}\text { Analytical } \\
\text { Lines } \\
\text { (nm) }\end{array}$} & \multicolumn{3}{|c|}{ Spectral Interference from $\mathrm{Nd}$} & \multicolumn{4}{|c|}{ Analytical Performance in Presence of Nd } \\
\hline & & $\begin{array}{l}\text { Contribution } \\
\text { from } \\
1000 \mathrm{mg} / \mathrm{L} \mathrm{Nd}\end{array}$ & $\begin{array}{l}\text { Correction } \\
\text { Factor }\end{array}$ & $\begin{array}{l}\text { Tolerance } \\
\text { Level } \\
(\mathrm{mg} / \mathrm{L})\end{array}$ & $\begin{array}{c}\text { D.L. } \\
(\mathrm{mg} / \mathrm{L})\end{array}$ & $\begin{array}{l}\text { Sensitivity } \\
\text { (counts/ } \\
\mathrm{mg} / \mathrm{L}\end{array}$ & $\begin{array}{c}\text { Linear } \\
\text { Dynamic } \\
\text { Range }(\mathrm{mg} / \mathrm{L})\end{array}$ & $\%$ RSD \\
\hline \multirow[t]{4}{*}{$\mathrm{Pb}$} & 220.353 & 2 & $2.0 \times 10^{-3}$ & 10 & 0.12 & 275 & $0.12-850$ & 3.8 \\
\hline & 405.778 & 12.7 & $1.3 \times 10^{-2}$ & 1 & 0.13 & 777 & $0.13-950$ & 2.2 \\
\hline & 283.305 & 1.1 & $1.1 \times 10^{-3}$ & 10 & 0.068 & 719 & $0.068-950$ & 1.7 \\
\hline & 261.418 & 4.3 & $4.3 \times 10^{-3}$ & 0.05 & 0.28 & 99 & $0.28-700$ & 4.4 \\
\hline \multirow[t]{5}{*}{ Cs } & 418.660 & 8.7 & $8.7 \times 10^{-3}$ & 10 & 0.094 & 1899 & $0.094-900$ & 3.2 \\
\hline & 448.691 & $\#$ & - & - & 0.85 & 58.8 & $0.85-500$ & 4.6 \\
\hline & 413.765 & 4.3 & $4.3 \times 10^{-3}$ & 10 & 0.083 & 2312 & $0.083-1000$ & 1.8 \\
\hline & 413.380 & 6 & $6.0 \times 10^{-3}$ & 1 & 0.055 & 2314 & $0.055-1000$ & 1.2 \\
\hline & 393.373 & $\#$ & - & - & 0.21 & 2216 & $0.21-1000$ & 1.4 \\
\hline \multirow[t]{4}{*}{ In } & 230.606 & 1.3 & $1.3 \times 10^{-3}$ & 10 & 0.07 & 577 & $0.07-800$ & 4.3 \\
\hline & 325.609 & $\#$ & - & - & 0.074 & 1489 & $0.074-950$ & 2.8 \\
\hline & 451.131 & $\#$ & - & - & 8.4 & 31 & 8.4-900 & 3.6 \\
\hline & 303.936 & 12.7 & $1.3 \times 10^{-2}$ & 1 & 0.1 & 535 & $0.1-900$ & 3.5 \\
\hline \multirow[t]{3}{*}{$\mathrm{La}$} & 408.672 & 12 & $1.2 \times 10^{-2}$ & 0.5 & 0.012 & 5525 & $0.5-1000$ & 1.1 \\
\hline & 333.749 & 5.7 & $5.7 \times 10^{-3}$ & 0.05 & 0.017 & 3927 & $0.017-1000$ & 1.6 \\
\hline & 379.478 & 6.6 & $6.6 \times 10^{-3}$ & 1 & 0.013 & 5142 & $0.013-1000$ & 1.0 \\
\hline \multirow[t]{4}{*}{$\operatorname{Pr}$} & 411.846 & 5.2 & $5.2 \times 10^{-3}$ & 10 & 0.033 & 3257 & $0.033-1000$ & 1.1 \\
\hline & 417.939 & 20 & $2.0 \times 10^{-2}$ & 0.05 & 0.038 & 3605 & $0.038-1000$ & 1.2 \\
\hline & 422.535 & 2.3 & $2.3 \times 10^{-3}$ & 10 & 0.034 & 2983 & $0.034-1000$ & 1.4 \\
\hline & 414.311 & 11.6 & $1.2 \times 10^{-2}$ & 1 & 0.019 & 4095 & $0.109-1000$ & 1.0 \\
\hline
\end{tabular}

Note: \# significant interference.

- Synthetic sample 7 (S7): $0.2 \mathrm{mg} / \mathrm{L}$ of $\mathrm{Ag}, \mathrm{B}, \mathrm{Ba}, \mathrm{Ca}, \mathrm{Cd}, \mathrm{Co}$, $\mathrm{Cu}, \mathrm{Fe}, \mathrm{Mg}, \mathrm{Na}, \mathrm{Sr}, \mathrm{Zn}, \mathrm{Gd}$ and $\mathrm{Lu}$ in $1000 \mathrm{mg} / \mathrm{L}$ of $\mathrm{Nd}$

- Synthetic sample 8 (S8): $2 \mathrm{mg} / \mathrm{L}$ of $\mathrm{Ag}, \mathrm{B}, \mathrm{Ba}, \mathrm{Ca}, \mathrm{Cd}, \mathrm{Co}$, $\mathrm{Cu}, \mathrm{Fe}, \mathrm{Mg}, \mathrm{Na}, \mathrm{Sr}, \mathrm{Zn}, \mathrm{Gd}$ and $\mathrm{Lu}$ in $1000 \mathrm{mg} / \mathrm{L}$ of $\mathrm{Nd}$

The analysis of these samples revealed that $0.1 \mathrm{mg} / \mathrm{L}$ of $\mathrm{Bi}, \mathrm{Li}, \mathrm{Mn}$, $\mathrm{Cr} ; 0.6 \mathrm{mg} / \mathrm{L}$ of $\mathrm{Ga}, \mathrm{Ni}, \mathrm{Al}, \mathrm{Tl}$, and Dy; $0.2 \mathrm{mg} / \mathrm{L}$ of $\mathrm{Ag}, \mathrm{B}, \mathrm{Ba}, \mathrm{Ca}, \mathrm{Cd}$, $\mathrm{Co}, \mathrm{Cu}, \mathrm{Fe}, \mathrm{Mg}, \mathrm{Na}, \mathrm{Sr}, \mathrm{Zn}, \mathrm{Gd}$, and $\mathrm{Lu} ; 2 \mathrm{mg} / \mathrm{L}$ of $\mathrm{Pb}$, In, Pr; and $5 \mathrm{mg} / \mathrm{L}$ of Cs and La can be determined in a Nd matrix without any chemical separation. The analytical results are summarized in Table VIII and were found to be satisfactory.

\section{CONCLUSION}

A systematic study was performed on the spectral interference of $\mathrm{Nd}$ on 28 analytes involving identification of the least interfered analytical lines of these analytes, determination of their tolerance level, evaluation of the correction factors and analytical performance of these lines in the presence of a Nd matrix (i.e., detection limits, sensitivity, linear dynamic range, and \%RSD), Their most suitable analytical lines for their determination in a Nd matrix wer as follows: Ag $328.068 \mathrm{~nm}$, B $208.959 \mathrm{~nm}$, Ba $455.404 \mathrm{~nm}$, Ca $393.366 \mathrm{~nm}$, Cd $226.502 \mathrm{~nm}$, Co $238.892 \mathrm{~nm}$, $\mathrm{Cu} 324.754 \mathrm{~nm}$, Fe $239.562 \mathrm{~nm}$, $\mathrm{Mg} 279.553 \mathrm{~nm}, \mathrm{Na} 588.995 \mathrm{~nm}$, Sr 421.552 nm, Zn 213.856 nm,
Gd $342.247 \mathrm{~nm}, \mathrm{Lu} 261.542 \mathrm{~nm}$ $\mathrm{Pb} 283.305 \mathrm{~nm}$, In $230.606 \mathrm{~nm}$, Cs $413.380 \mathrm{~nm}$ and $413.765 \mathrm{~nm}$, La $333.749 \mathrm{~nm}, \operatorname{Pr} 422.535 \mathrm{~nm}$, Ga 417.206 nm, Ni 231.604 nm, Al 308.215 nm, Tl $190.864 \mathrm{~nm}$, Dy $340.780 \mathrm{~nm}$, Bi $306.772 \mathrm{~nm}$, Li $670.780 \mathrm{~nm}$, Mn $257.611 \mathrm{~nm}$, and $\mathrm{Cr} 284.325 \mathrm{~nm}$.

An ICP-AES-based simultaneous determination of $\mathrm{Nd}$ revealed that though all of the chosen analytical lines, i.e., $401.225 \mathrm{~nm}, 406.109 \mathrm{~nm}$, $417.731 \mathrm{~nm}$, and $430.358 \mathrm{~nm}$ can be suitably used for the determination of $\mathrm{Nd}$, the best line is $\mathrm{Nd}$ $401.225 \mathrm{~nm}$. Using the abovementioned most suitable lines of the analytes, they can be determined at trace levels in a Nd matrix without any chemical separation. 
TABLE VIII

Determination of the Analytes (a) Bi, Li, Mn, Cr; (b) Ga, Ni, Al, T1 and Dy; (c) Pb, In, Ce, La, Pr; and (d) Ag, B, Ba, Ca, Cd, Co, Cu, Fe, Mg, Na, Sr, Zn, Gd and Lu in Nd Matrix Without Chemical Separation Using Synthetic Samples

\begin{tabular}{|c|c|c|c|c|c|c|c|}
\hline \multirow[b]{2}{*}{ Elements } & \multirow{2}{*}{\multicolumn{2}{|c|}{$\begin{array}{l}\text { Analytical Lines } \\
\text { (nm) }\end{array}$}} & \multicolumn{2}{|c|}{ S1 } & \multicolumn{3}{|c|}{ S2 } \\
\hline & & & \multicolumn{2}{|c|}{\begin{tabular}{|cc}
$\begin{array}{c}\text { Actual Concentration } \\
(\mathrm{mg} / \mathrm{L})\end{array}$ & $\begin{array}{c}\text { Amount Estimated } \\
(\mathrm{mg} / \mathrm{L})\end{array}$ \\
\end{tabular}} & \multicolumn{3}{|c|}{\begin{tabular}{|cc} 
Actual Concentration & Amount Estimated \\
$(\mathrm{mg} / \mathrm{L})$ & $(\mathrm{mg} / \mathrm{L})$
\end{tabular}} \\
\hline $\mathrm{Bi}$ & \multirow{4}{*}{\multicolumn{2}{|c|}{$\begin{array}{l}306.772 \\
670.780 \\
257.611 \\
284.325\end{array}$}} & \multicolumn{2}{|r|}{$0.097 \pm 0.004$} & \multicolumn{2}{|r|}{1} & $0.97 \pm 0.05$ \\
\hline $\mathrm{Li}$ & & & \multicolumn{2}{|r|}{$0.010 \pm 0.005$} & \multicolumn{2}{|r|}{1} & $0.94 \pm 0.08$ \\
\hline Mn & & & \multicolumn{2}{|r|}{$0.098 \pm 0.005$} & \multicolumn{2}{|r|}{1} & $1.07 \pm 0.06$ \\
\hline $\mathrm{Cr}$ & & & \multicolumn{2}{|r|}{$0.099 \pm 0.006$} & \multicolumn{2}{|r|}{1} & $1.04 \pm 0.05$ \\
\hline Elements & $\begin{array}{l}\text { Analytical } \\
\text { Lines } \\
(\mathrm{nm})\end{array}$ & \multicolumn{3}{|c|}{ 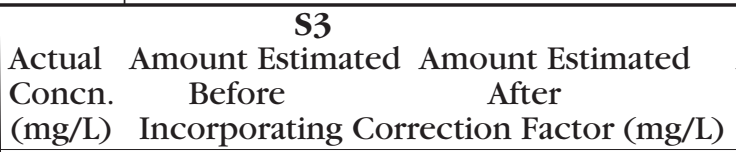 } & $\begin{array}{r}\text { Actual } \\
\text { Conc. } \\
(\mathrm{mg} / \mathrm{L})\end{array}$ & $\begin{array}{c}\mathbf{S 4} \\
\text { Amount Estimate } \\
\text { Before } \\
\text { Incorporating Cor }\end{array}$ & $\begin{array}{l}\text { d Amount Estimated } \\
\text { After } \\
\text { rection Factor }(\mathrm{mg} / \mathrm{L})\end{array}$ \\
\hline $\mathrm{Ga}$ & 417.206 & 0.6 & $1.32 \pm 0.04$ & 0.56 & 2 & $2.80 \pm 0.09$ & 2.04 \\
\hline $\mathrm{Ni}$ & 231.604 & 0.6 & $1.30 \pm 0.05$ & 0.61 & 2 & $2.70 \pm 0.11$ & 2.01 \\
\hline $\mathrm{Al}$ & 308.215 & 0.6 & $1.18 \pm 0.06$ & 0.58 & 2 & $2.58 \pm 0.13$ & 1.98 \\
\hline $\mathrm{Tl}$ & 190.864 & 0.6 & $1.30 \pm 0.03$ & 0.61 & 2 & $2.65 \pm 0.07$ & 1.96 \\
\hline Dy & 340.780 & 0.6 & $1.09 \pm 0.04$ & 0.65 & 2 & $2.42 \pm 0.08$ & 1.98 \\
\hline Elements & $\begin{array}{l}\text { Analytical } \\
\text { Lines } \\
(\mathrm{nm})\end{array}$ & $\begin{array}{l}\text { Actual } \\
\text { Concn. } \\
(\mathrm{mg} / \mathrm{L})\end{array}$ & $\begin{array}{l}\text { S5 } \\
\text { Amount Estimated An } \\
\text { Before } \\
\text { Incorporating Correct }\end{array}$ & $\begin{array}{l}\text { nount Estimated } \\
\text { After } \\
\text { ion Factor }(\mathrm{mg} / \mathrm{L})\end{array}$ & $\begin{array}{l}\text { Actual } \\
\text { Conc. } \\
(\mathrm{mg} / \mathrm{L})\end{array}$ & $\begin{array}{c}\text { S6 } \\
\text { Amount Estimate } \\
\text { Before } \\
\text { Incorporating Cor }\end{array}$ & $\begin{array}{l}\text { ed Amount Estimated } \\
\text { After } \\
\text { rection Factor (mg/L) }\end{array}$ \\
\hline $\mathrm{Pb}$ & 283.305 & 2 & $3.0 \pm 0.2$ & 1.9 & 5 & $6.0 \pm 0.3$ & 4.9 \\
\hline In & 230.606 & 2 & $3.5 \pm 0.3$ & 2.2 & 5 & $6.1 \pm 0.2$ & 4.8 \\
\hline Cs & 413.380 & 5 & $9.4 \pm 0.4$ & 5.1 & 10 & $14.6 \pm 0.6$ & 10.3 \\
\hline & 413.765 & 5 & $9.4 \pm 0.4$ & 4.8 & 10 & $15.0 \pm 0.7$ & 10.4 \\
\hline $\mathrm{La}$ & 333.749 & 5 & $10.9 \pm 0.5$ & 5.8 & 10 & $15.5 \pm 0.8$ & 9.8 \\
\hline $\operatorname{Pr}$ & 422.535 & 2 & $4.1 \pm 0.2$ & 1.8 & 5 & $7.2 \pm 0.3$ & 4.9 \\
\hline Elements & $\begin{array}{l}\text { Analytical } \\
\text { Lines } \\
\text { (nm) }\end{array}$ & $\begin{array}{l}\text { Actual } \\
\text { Concn. } \\
(\mathrm{mg} / \mathrm{L})\end{array}$ & $\begin{array}{l}\text { S7 } \\
\text { Amount Estimated An } \\
\text { Before } \\
\text { Incorporating Correct }\end{array}$ & $\begin{array}{l}\text { nount Estimated } \\
\text { After } \\
\text { ion Factor }(\mathrm{mg} / \mathrm{L})\end{array}$ & $\begin{array}{r}\text { Actual } \\
\text { Conc. } \\
(\mathrm{mg} / \mathrm{L})\end{array}$ & $\begin{array}{c}\mathbf{S 8} \\
\text { Amount Estimat } \\
\text { Before } \\
\text { Incorporating Cor }\end{array}$ & $\begin{array}{l}\text { ed Amount Estimated } \\
\text { After } \\
\text { rection Factor }(\mathrm{mg} / \mathrm{L})\end{array}$ \\
\hline Ag & 328.068 & 0.2 & $0.52 \pm 0.02$ & 0.18 & 2 & $2.36 \pm 0.08$ & 2.02 \\
\hline $\mathrm{B}$ & 208.959 & 0.2 & $0.32 \pm 0.01$ & 0.22 & 2 & $2.00 \pm 0.09$ & 1.90 \\
\hline $\mathrm{Ba}$ & 455.404 & 0.2 & $0.55 \pm 0.02$ & 0.21 & 2 & $2.35 \pm 0.11$ & 2.01 \\
\hline $\mathrm{Ca}$ & 393.366 & 0.2 & $0.50 \pm 0.03$ & 0.18 & 2 & $2.30 \pm 0.12$ & 1.98 \\
\hline $\mathrm{Cd}$ & 226.502 & 0.2 & $0.35 \pm 0.01$ & 0.22 & 2 & $2.09 \pm 0.08$ & 1.96 \\
\hline Co & 238.892 & 0.2 & $0.52 \pm 0.02$ & 0.19 & 2 & $2.29 \pm 0.07$ & 1.96 \\
\hline $\mathrm{Cu}$ & 324.754 & 0.2 & $0.45 \pm 0.03$ & 0.23 & 2 & $2.35 \pm 0.09$ & 2.03 \\
\hline $\mathrm{Fe}$ & 239.562 & 0.2 & $0.50 \pm 0.02$ & 0.19 & 2 & $2.30 \pm 0.08$ & 1.99 \\
\hline $\mathrm{Mg}$ & 279.553 & 0.2 & $0.30 \pm 0.03$ & 0.20 & 2 & $2.11 \pm 0.09$ & 2.01 \\
\hline $\mathrm{Na}$ & 588.995 & 0.2 & $0.35 \pm 0.03$ & 0.21 & 2 & $2.05 \pm 0.08$ & 2.01 \\
\hline $\mathrm{Sr}$ & 421.552 & 0.2 & $0.30 \pm 0.02$ & 0.21 & 2 & $2.11 \pm 0.08$ & 2.02 \\
\hline $\mathrm{Zn}$ & 213.856 & 0.2 & $0.32 \pm 0.02$ & 0.23 & 2 & $2.15 \pm 0.10$ & 1.96 \\
\hline Gd & 342.247 & 0.2 & $0.58 \pm 0.01$ & 0.22 & 2 & $2.34 \pm 0.10$ & 1.98 \\
\hline $\mathrm{Lu}$ & 261.542 & 0.2 & $0.33 \pm 0.01$ & 0.19 & 2 & $2.08 \pm 0.13$ & 1.94 \\
\hline
\end{tabular}




\section{A $_{\text {Spectroscopy }}^{\text {pectros }}$ \\ 1 Vol. 36(1), Jan./Feb. 2015}

\section{ACKNOWLEDGMENT}

The authors wish to acknowledge the constant support of Dr. A. Goswami, Head, Radiochemistry Division, throughout this study.

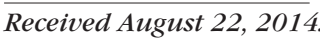

\section{REFERENCES}

1. S. I. Prokopchuk, E.V. Smrinova and N.G. Balbekina, J Anal. Chem. 55, 313 (2000).

2. P.W.J.M. Bouman's and J.J.A.M. Vrakking, Spectrochim. Acta 39B, 1239 (1984)

3. J. P. Robin, Pro. Anal. Atom Spectrosc, 5, 79 (1982).

4. M.H. Abdallah, J. M. Mermet and C. Trassy, Anal. Chim. Acta 87, 329 (1976).

5. A. Mazzucotellit, F. De Paz, E. Magi and R. Frache, Anal. Sciences 8189 (1992).

6. G. Sheng, Y. Hai , Z. Zhigang, Z. Zhixia, Y. Pengyuan, W. Xiaoru,H. Benli and L. Bing, Spectrochim. Acta 52B, 1567 (1997).

7. N.E. Topp, The Chemistry of rare earth element. Elsevier, NY, USA (1965).

8. T. Kano and H. Yanagida, Rare earths: properties and applications, J.H. Jolly, Rare Earth elements and Yttrium; Mineral facts and problems, Bureau of mines, U.S. Department of the interior, Washington, DC, USA (1975).

10. Preston, J.S., Hydrometallurgy 42, 151 (1966)

11. J. Broekaert, F Leis and K Laqua, Spectrochim Acta 34B, 73 (1979).

12. I. Brenner, A. Watson, T. Steele, E. Jones and M Goncalves, Spectrochim. Acta 36B, 785 (1981).

13. J. Crock and F. Lichte, Anal. Chem. 54, 1329 (1982).

14. A Bolton, J Hwang and A. Voet, Spectrochim. Acta, 38B, 165 (1983).

15. S. Buchanan and L. Dale, Spectrochim. Acta 41B, 237 (1986).
16. I. Roelandts, At. Spectrosc. 9, 49 (1988).

17. S. Greenfield, M. S. Salman and J.F. Tyson, Spectrochim. Acta 43B, 1087 (1988).

18. LuísCláudio de Oliveira1, Ieda de Souza Silva and M Isabel Rucandio, Rare earth elements determination by ICP-OES in high purity gadolinium, International Nuclear Atlantic Conference - INAC 2009, Rio de Janeiro, RJ, Brazil, ASSOCIAÇÃO BRASILEIRA DE ENERGIA NUCLEAR - ABEN, ISBN: 978-8599141-03-8 (2009).

19. I. Hajime and Satoh, Talanta 29, 243 (1982).

20. M. I. Rucandio, Fresenius J. Anal. Chem. 357, 661 (1997).

21. S. Greenfield, I.LI. Jones and C.T. Berry, Analyst 89, 713 (1964).

22. R.H. Wendt and V.A. Fassel, Anal. Chem. 37920 (1965).

23. S. F. Marsh, Anal. Chem. 39, 641 (1967).

24. J. J. McCown and R. P. Larsen, Anal. Chem. 33, 1003 (1961).

25. A. Masuda, N. Nakamura and T. Tanaka, Geochim. Cosmochim. Acta 37, 239 (1973).

26. V.A. Fassel and R.N. Kniseley, Anal. Chem. 46, 1110A (1974).

27. V.C. Adya, A.Sengupta, S.V. Godbole, At. Spectrosc. 35(1), 25 (2014).

28. A.Sengupta, V.C.Adya, T.K. Seshagiri, and S.V. Godbole, At. Spectrosc. 34(2), 53 (2013).

29. A.Sengupta and V.C.Adya, At. Spectrosc. 34(6), 207 (2013).

30. A. Sengupta, S.K. Thulasidas and V. Natarajan, At. Spectrosc. 35(6), 2472014 . 